Correlation between temperature dependence of Raman shifts and in-plane strains in an AIGaN/GaN stack

Daisuke Kosemura, Vice Sodan, and Ingrid De Wolf

Citation: J. Appl. Phys. 121, 035702 (2017); doi: 10.1063/1.4974366

View online: http://dx.doi.org/10.1063/1.4974366

View Table of Contents: http://aip.scitation.org/toc/jap/121/3

Published by the American Institute of Physics 


\title{
Correlation between temperature dependence of Raman shifts and in-plane strains in an AIGaN/GaN stack
}

\author{
Daisuke Kosemura, ${ }^{1, a)}$ Vice Sodan, ${ }^{1,2}$ and Ingrid De Wolf ${ }^{1,2, a)}$ \\ ${ }^{1}$ imec, Kapeldreef 75, 3001 Leuven, Belgium \\ ${ }^{2}$ Department of Materials Engineering, Katholieke Universiteit Leuven, 3000 Leuven, Belgium
}

(Received 1 October 2016; accepted 7 January 2017; published online 19 January 2017)

\begin{abstract}
The temperature dependence of Raman shifts for different layers and different optical phonon modes in an $\mathrm{AlGaN} / \mathrm{GaN}$ stack was examined in this study. The slopes of the Raman shifts as a function of temperature for the $\mathrm{GaN}$ and $\mathrm{Al}_{x} \mathrm{GaN}$ layers were found to vary, especially for the $\mathrm{E}_{2}$ high mode compared with that for the $\mathrm{A}_{1}$ (LO) mode. To further investigate these fluctuations in the temperature dependence of Raman shifts, a detailed evaluation was conducted for the depth distribution of in-plane strains in the $\mathrm{AlGaN} / \mathrm{GaN}$ stack by detecting each of the layers simultaneously in a single Raman spectrum. The temperature dependence fluctuations for the $\mathrm{E}_{2}$ high modes of the $\mathrm{Al}_{x} \mathrm{GaN}$ layers are considered to be related to the in-plane strain distribution with depth. Published by AIP Publishing. [http://dx.doi.org/10.1063/1.4974366]
\end{abstract}

\section{INTRODUCTION}

The self-heating effect of $\mathrm{AlGaN} / \mathrm{GaN}$ high electron mobility transistors (HEMTs) is an important factor that plays a role in limiting their performance. ${ }^{1-3}$ Moreover, the elevated temperatures associated with Joule heating at high power densities pose a reliability issue; therefore, it is important to monitor the temperatures in the $\mathrm{GaN}$ transistors to explore all benefits of GaN-based devices. Several techniques based on electrical measurements, physical contact, optical measurements, and simulations have been proposed to achieve this. ${ }^{1,4-6}$ In our previous study, we used four electrical methods and one optical method, Raman spectroscopy, for temperature sensing in AlGaN/GaN HEMTs, and the results indicated large variations among the temperature values obtained using each method. ${ }^{1}$ Furthermore, a combination of technology computer aided design and finite element method simulation was implemented to validate the experimental results, and it was found that the simulation was able to explain the differences. However, further investigation is required to understand the relationship between the device performance and temperature.

The AlGaN/GaN HEMT structures tend to become complex to form a high quality GaN channel layer where a twodimensional electron gas is generated. Such complex structures are formed owing to a large lattice mismatch between GaN and commonly used (111)-oriented $\mathrm{Si}$ substrate. Fig. 1(a) shows a schematic of the sample that was used in this study. As shown in this figure, an AlGaN/GaN interface with a 3-nm GaN cap layer is followed by several $\mathrm{AlGaN}$ layers including an AlN buffer layer to mitigate the lattice mismatch between $\mathrm{GaN}$ and $\mathrm{Si}$.

The presence of defects and strains in the buffer layers, the thicknesses of which are sometimes thin, can cause the physical properties of the wurtzite structured $\mathrm{AlGaN}$ to differ from those of the bulk material. For instance, in Ref. 2, the

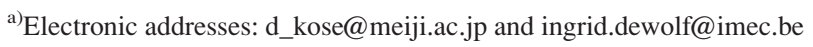

authors reported that the thermal conductivity of a $150-\mathrm{nm}$ thick GaN film was approximately $60 \mathrm{~W} \mathrm{~m}^{-1} \mathrm{~K}^{-1}$, which was lower than that of the bulk material by more than $50 \%$. For a comprehensive interpretation of HEMT operations, each AlGaN-comprising buffer layer should be evaluated simultaneously with the GaN channel layer.

Useful information about the physical properties of wurtzite-structured III-nitride materials can be determined from their phonon behavior. ${ }^{7-11}$ In this study, we have investigated the temperature dependence of Raman shifts for different layers and different optical phonon modes in an $\mathrm{AlGaN} / \mathrm{GaN}$ stack to obtain the relationship between the phonon behavior and temperature, which enables us to consider the thermal properties of each layer for use in designing the $\mathrm{AlGaN} / \mathrm{GaN}$ stack. In addition, the in-plane strain induced in the $\mathrm{AlGaN} / \mathrm{GaN}$ stack was calculated from the Raman wavenumber shifts at room temperature (RT) using the isotropic biaxial stress coefficient to further understand the temperature dependence of the phonon modes.

\section{EXPERIMENTAL PROCEDURE}

Fig. 1(a) shows a schematic of the $\mathrm{AlGaN} / \mathrm{GaN}$ stack structure of the sample used in this study. Buffer layers containing $\mathrm{AlN}$ and $8 \%, 44 \%$, and $75 \% \mathrm{Al}_{x} \mathrm{GaN}$ were deposited on a (111)-oriented Si substrate to mitigate the lattice mismatch between $\mathrm{GaN}$ and $\mathrm{Si}$. A 150-nm-thick GaN channel layer with 15 -nm-thick $20 \% \mathrm{Al}_{x} \mathrm{GaN}$ barrier and a 3-nmthick GaN cap layers is present above the buffer layers. These AlGaN layers have a wurtzite crystal structure.

For the Raman measurements, an excitation laser with a wavelength of $532 \mathrm{~nm}$, which corresponds to an energy of approximately $2.33 \mathrm{eV}$, was used. The absorption coefficient of $\mathrm{GaN}$ is approximately zero at this energy level because the direct band gap energy is approximately $3.4 \mathrm{eV} .{ }^{12}$ The direct band gap of AlN is approximately $6.2 \mathrm{eV},{ }^{13}$ and the band gap of $\mathrm{Al}_{x} \mathrm{Ga}_{1-x} \mathrm{~N}$ eutectic compounds ranges between 3.4 and $6.2 \mathrm{eV}$. This is the reason for the laser light being 
3-nm GaN

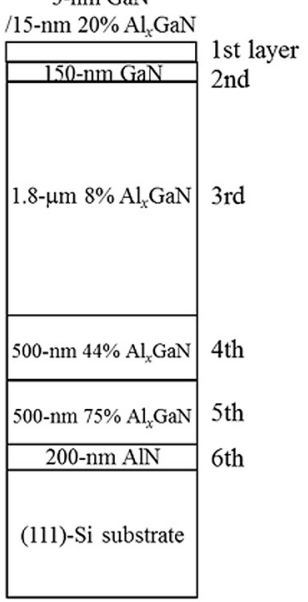

(a)

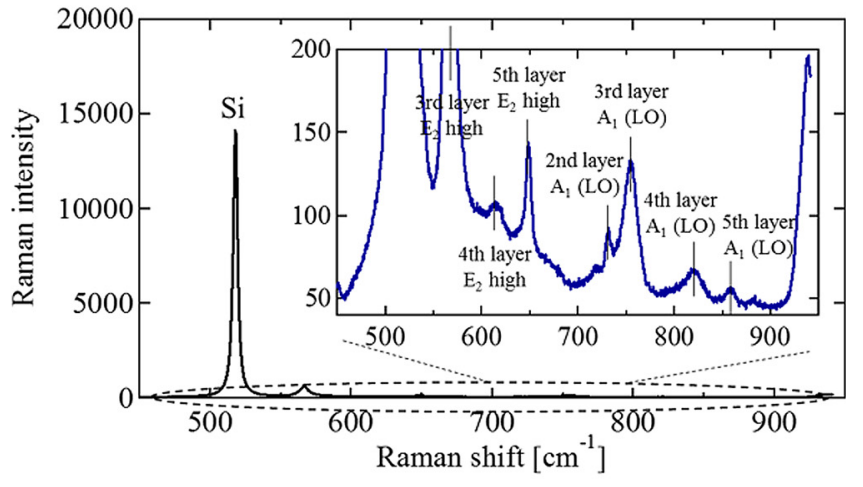

(b) transmitted through the entire AlGaN stack and being absorbed in the Si substrate, through which the phonon information of each layer can be obtained.

The polarization configuration is $z(x, x) \bar{z}$ or $\mathrm{z}(\mathrm{y}, \mathrm{y}) \bar{z}$ in the Porto notation, where the coordinates were defined as $x$ : [21 $\overline{1} 0]$ ( $a$ direction), $y:$ [01 10] ( $m$ direction), and $z$ : [0001] ( $c$ direction). According to the group theory, because a wurtzite structure belongs to the $\mathrm{C}_{6 v}{ }^{4}$ space group, the optical phonon modes $2 \mathrm{E}_{2}\left(\mathrm{E}_{2}\right.$ high and low) and $\mathrm{A}_{1}(\mathrm{LO})$ are expected to get excited out of the six Raman-active 1st-order optical phonon modes, $2 \mathrm{E}_{2}+1 \mathrm{~A}_{1}(\mathrm{LO}$ and $\mathrm{TO})+1 \mathrm{E}_{1}(\mathrm{LO}$ and TO). ${ }^{7,14-18}$ The $E_{2}$ high and $A_{1}$ (LO) modes were used in this study to examine the phonon behavior in the AlGaN stack, as the $\mathrm{E}_{2}$ low mode is generally not used for analysis because of its low intensity.

The sample was heated at different temperatures starting from ambient temperature up to $240{ }^{\circ} \mathrm{C}$, and the peak positions of $\mathrm{E}_{2}$ high and $\mathrm{A}_{1}(\mathrm{LO})$ were monitored as a function of the backplate temperature in steps of $30^{\circ} \mathrm{C}$. The $\mathrm{Si}$ peak was also monitored and used as a wavenumber correction at each temperature. A conductive paste was used to improve heat transfer between the heater and the sample. Two different levels of laser power were applied to ensure that the sample heating effect was negligible. The laser power after the objective lens was measured to be $520 \mu \mathrm{W}$ and was reduced by a factor of 1/4 using an attenuation filter. During the heating, an objective lens with a longworking distance of $18 \mathrm{~mm}$ was used to avoid damage to the objective lens, the magnification and numerical aperture of which were $50 \times$ and 0.35 , respectively. Three sets of measurements were thus conducted: one without the conductive paste at a high laser power (data 1), one with the conductive paste at a high laser power (data 2), and one without conductive paste at a low laser power (data 3 ).

Two methods are available for measuring the temperature of the materials from the obtained Raman spectra. The first one is to measure the temperature at the peak position and the second one is to measure it using the intensity ratio between the Stokes and anti-Stokes peaks. However, the first method provides a more accurate temperature value because the intensity ratio can change with the change in material properties, such as reflectivity and permittivity, during material heating. ${ }^{19}$

\section{RESULTS AND DISCUSSION}

Fig. 1(b) shows a Raman spectrum of an AlGaN/GaN stack on a (111)-oriented Si substrate obtained at RT, with a very strong peak from the Si substrate. The signal from the AlGaN stack is weak compared with that from the Si substrate; therefore, the Raman intensity scale is limited, as shown in the inset of Fig. 1(b). In the inset figure, the $\mathrm{E}_{2}$ high peaks from the $8 \%, 44 \%$, and $75 \% \mathrm{Al}_{x} \mathrm{GaN}$ layers are visible, whereas those from the GaN cap and $\mathrm{AlGaN}$ barrier layers are not seen because these layers $(3 \mathrm{~nm}$ and $15 \mathrm{~nm}$, respectively) are too thin to be detected. The $\mathrm{E}_{2}$ high peak from the 150-nm GaN channel layer cannot be seen in the spectrum because the peak positions between the GaN channel and $8 \% \mathrm{Al}_{x} \mathrm{GaN}$ layers are too close to be distinguished. The 200-nm-thick AlN layer is expected to be detected in the spectrum, but this layer is located too deep in the structure for an adequate amount of Raman scattering to be collected. The signal from the deep layer easily becomes weak because of reflections at the interfaces of each layer. That is why the Raman scattering from the thin AlN layer cannot be observed although that from $\mathrm{Si}$ with a large scattering volume can still be observed.

In contrast, the $\mathrm{A}_{1}(\mathrm{LO})$ peaks from the GaN channel layer and $8 \%, 44 \%$, and $75 \% \mathrm{Al}_{x} \mathrm{GaN}$ layers were detected, as shown in the inset of Fig. 1(b). The reason for the missing peaks from the GaN cap, the AlGaN barrier, and the AlN layers is the same as that for the $\mathrm{E}_{2}$ high mode. Therefore, the temperature dependence of Raman shifts for different layers and different phonon modes can be simultaneously evaluated, and more information can be derived from this spectrum.

The $\mathrm{E}_{2}$ high mode is observed to have the highest peak in the $8 \% \mathrm{Al}_{x} \mathrm{GaN}$ layer because it has the largest scattering volume with a thickness of $1.8 \mu \mathrm{m}$. Significant broadening is observed in the $44 \% \mathrm{Al}_{x} \mathrm{GaN}$ peak owing to its mid-range $\mathrm{Al}$ concentration. Both GaN-like and AlN-like $\mathrm{E}_{2}$ high modes 
have been reported to occur together for such compositions. ${ }^{20,21}$ In fact, the full width at half maximum of the peak for the $44 \% \mathrm{Al}_{x} \mathrm{GaN}$ layer was $16.4 \mathrm{~cm}^{-1}$, whereas it was 7.8 and $6.2 \mathrm{~cm}^{-1}$ for the $8 \%$ and $75 \% \mathrm{Al}_{x} \mathrm{GaN}$ layers, respectively. Therefore, an AlN-like peak can be considered to have begun appearing in the $44 \% \mathrm{Al}_{x} \mathrm{GaN}$ spectrum, whereas the peak from the $75 \% \mathrm{Al}_{x} \mathrm{GaN}$ spectrum is clear and sharp, indicating that the AlN-like $\mathrm{E}_{2}$ high mode peak is dominant and not mixed with the GaN-like peak.

The $\mathrm{A}_{1}$ (LO) peaks from the $\mathrm{GaN}$ channel and $8 \% \mathrm{Al}_{x} \mathrm{GaN}$ layers are clearly distinct because of the higher sensitivity of these peaks than that of the $\mathrm{E}_{2}$ high peak to $\mathrm{Al}$ concentration. The $\mathrm{A}_{1}(\mathrm{LO})$ peak from the $44 \% \mathrm{Al}_{x} \mathrm{GaN}$ layer exhibits broadening on the left side. This can be explained by a spatial correlation model as follows: The phonon dispersion of GaN or AlN spreads toward lower frequencies starting from the $\Gamma$ point of the Brillouin zone, ${ }^{8,18,22}$ and a lower-frequency peak appears if the momentum conservation rules break, which can occur in compound materials with a certain degree of lattice-disorder. ${ }^{20}$ In addition, the splitting seen for the $E_{2}$ high mode is not observed for $A_{1}$ (LO). ${ }^{20,21}$ The $\mathrm{A}_{1}$ (LO) peak from the $75 \% \mathrm{Al}_{x} \mathrm{GaN}$ layer was also detected, as shown in the inset of Fig. 1(b), and the line width of the $A_{1}(\mathrm{LO})$ peak in this layer is smaller than that of $44 \% \mathrm{Al}_{x} \mathrm{GaN}$ layer because of a lower degree of lattice-disorder. As described above, these peaks were clearly identified and monitored during the heating process to determine the temperature dependence of the phonon peak positions for the GaN channel layer and the $8 \%, 44 \%$, and $75 \% \mathrm{Al}_{x} \mathrm{GaN}$ layers.

Fig. 2 shows the Raman shifts of both the $\mathrm{E}_{2}$ high and $\mathrm{A}_{1}$ (LO) modes for $\mathrm{GaN}$ and $8 \%, 44 \%$, and $75 \% \mathrm{Al}_{x} \mathrm{GaN}$ as a function of temperature from RT to $240{ }^{\circ} \mathrm{C}$ although the $\mathrm{E}_{2}$ high mode for $\mathrm{GaN}$ is not present, as explained for Fig. 1. Changes in phonon behavior with temperature can generally be described by anharmonic effects. ${ }^{19,23-33}$ In addition, since our sample is an AlGaN stack rather than a bulk material, the thermal and lattice mismatches between different layers and the Si substrate also need to be accounted for using the following expression: ${ }^{23}$

$$
\omega(T)=\omega_{0}+\Delta \omega_{e}(T)+\Delta \omega_{d}(T)+\Delta \omega_{s}(T),
$$

where $\omega_{0}$ is the bulk value of phonon frequency at $0 \mathrm{~K}$, $\Delta \omega_{e}(T)$ is the shift due to thermal expansion of the material, $\Delta \omega_{d}(T)$ is the shift due to phonon-phonon interactions, which causes anharmonicity of the phonon behavior with temperature, and $\Delta \omega_{s}(T)$ is the shift due to thermal and lattice mismatches between different layers and the Si substrate. ${ }^{23}$

Cui et al. suggested applying a purely empirical equation to reproduce the temperature dependence of the Raman shifts, which is based on electron-phonon interactions of the band gap in the Einstein approximation ${ }^{19}$

$$
\omega(T)=\omega_{0}-\frac{C}{e^{D \hbar \omega_{0} / k_{B} T}-1},
$$

where $C$ and $D$ are fitting parameters. The study mentioned above claimed that Eq. (2) fits the experimental data better than the formula represented by the phonon-phonon interactions considering cubic and quartic terms (third- and fourth-order processes, respectively), i.e., the electron-phonon interaction is also important for expressing the phonon frequency behavior with temperature. ${ }^{19}$ The fitting curves calculated using Eq. (2) are shown in Fig. 2. In the temperature range from $\mathrm{RT}$ to $240^{\circ} \mathrm{C}$, the fitting curves obtained for the temperature dependence can be treated as linear, from which the slopes were extracted, as shown in Fig. 2..$^{23,25,26,30}$ Note that these slopes are valid for the $\mathrm{GaN}$ and $\mathrm{Al}_{x} \mathrm{GaN}$ layers of the stack used in this study, not for the bulk values.

Fig. 3 shows a comparison of the slopes obtained from the fitting curves for the temperature dependence of Raman shifts in $\mathrm{GaN}$ and $\mathrm{AlGaN}$ layers for the $\mathrm{E}_{2}$ high and $\mathrm{A}_{1}$ (LO) optical phonon modes. Large differences in slope are observed for each layer, especially for the $E_{2}$ high mode.
GaN
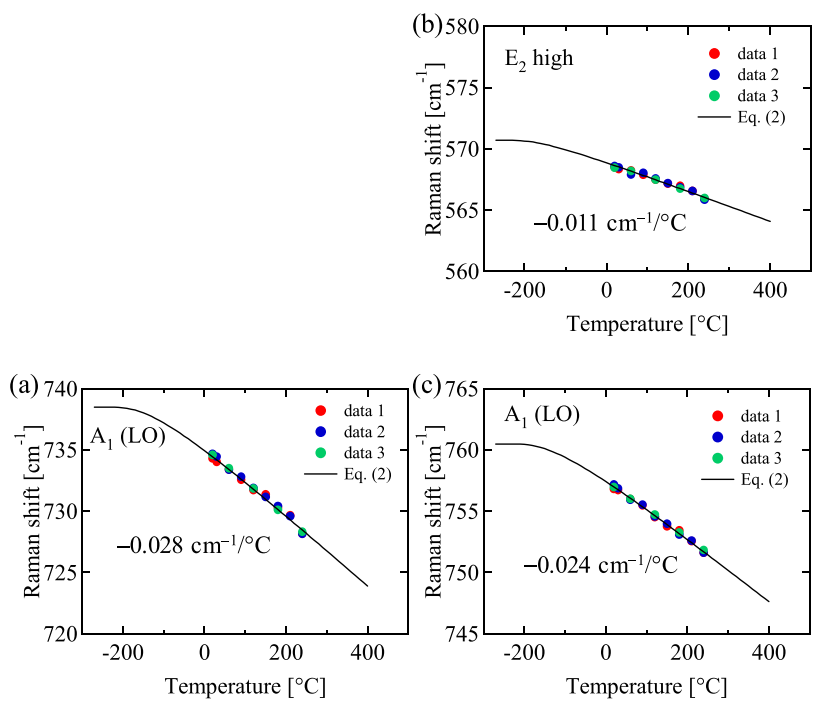
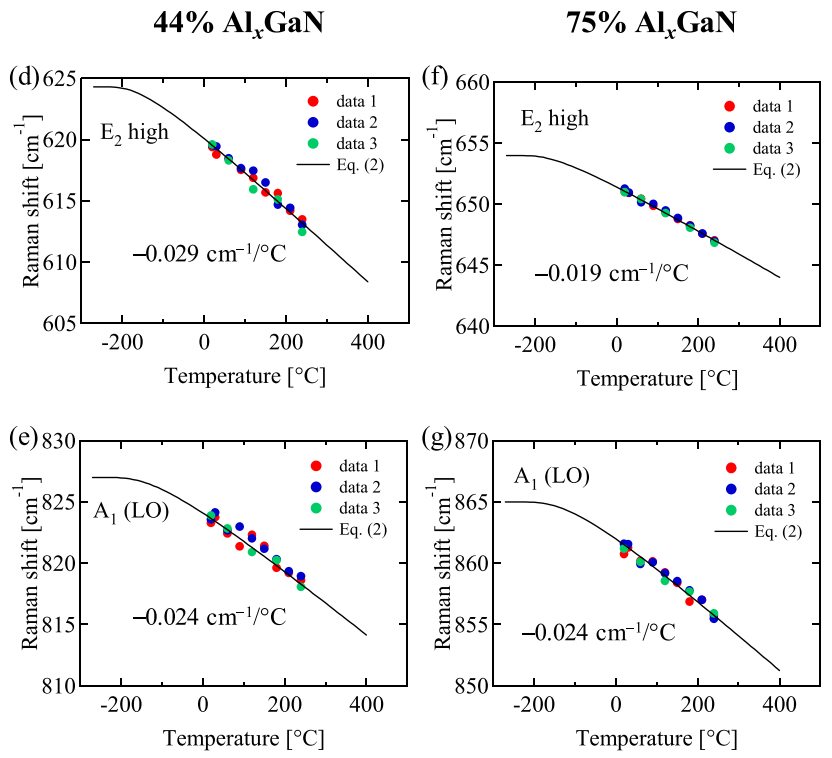

FIG. 2. Raman shifts of the $\mathrm{A}_{1}$ (LO) mode for (a) GaN and Raman shifts of both $\mathrm{E}_{2}$ high and $\mathrm{A}_{1}$ (LO) modes for ((b) and (c)) $8 \% \mathrm{Al}_{x} \mathrm{GaN}$, ((d) and (e)) $44 \%$ $\mathrm{Al}_{x} \mathrm{GaN}$, and ((f) and $\left.(\mathrm{g})\right) 75 \% \mathrm{Al}_{x} \mathrm{GaN}$ as a function of temperature from $\mathrm{RT}$ to $240{ }^{\circ} \mathrm{C}$. 


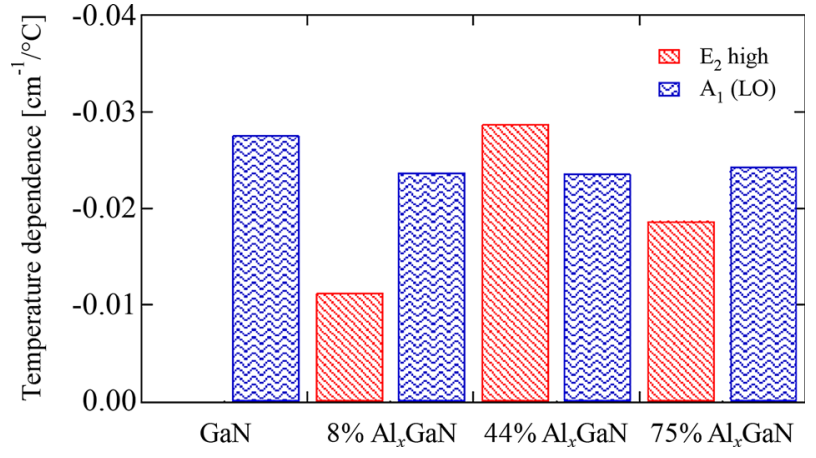

FIG. 3. Comparison of the slopes obtained from the fitting curves for the temperature dependence of Raman shifts in GaN and AlGaN layers for the $\mathrm{E}_{2}$ high and $\mathrm{A}_{1}(\mathrm{LO})$ optical phonon modes.

The polarizations of the $\mathrm{E}_{2}$ high and $\mathrm{A}_{1}(\mathrm{LO})$ modes are perpendicular and parallel to the $z$-axis, respectively, in our experimental configuration., ${ }^{9,34}$ A large in-plane strain is expected to result owing to the lattice mismatch between different $\mathrm{AlGaN}$ stack layers and the $\mathrm{Si}$ substrate, which may result in differences in the temperature dependence of phonon behavior, especially for the $\mathrm{E}_{2}$ high mode with a lateral polarization. Further details of this topic are as discussed below.

The in-plane strain was calculated from the Raman wavenumber shifts with respect to the strain-free frequency $\omega_{0}{ }^{\mathrm{RT}}$ at RT. The $\omega_{0}{ }^{\mathrm{RT}}\left(\mathrm{A}_{1} \mathrm{LO}\right)$ value of pure GaN was reported to be $734 \mathrm{~cm}^{-1} \cdot{ }^{20}$ On the other hand, to calculate $\omega_{0}{ }^{\mathrm{RT}}\left(\mathrm{A}_{1} \mathrm{LO}\right)$ for $\mathrm{Al}_{x} \mathrm{GaN}$ with a different $\mathrm{Al}$ concentration $x$, the following equation was proposed ${ }^{20}$

$$
\omega_{0}^{R T}\left(A_{1} \mathrm{LO}\right)=734+153 x+75 x(1-x) .
$$

As mentioned above, the $\mathrm{Al}$ concentration dependence of the $\mathrm{E}_{2}$ high mode is more complicated than that of the $\mathrm{A}_{1}$ (LO) mode because of the splitting phenomena; therefore, we used the peak position of $A_{1}$ (LO) and Eq. (3) for the in-plane strain calculation.

The Raman wavenumber shift $\Delta \omega$ for each GaN or AlGaN layer is shown in Table I. The in-plane stress, i.e., the isotropic biaxial stress $\sigma_{\text {biaxial, }}$ was calculated using the isotropic biaxial stress coefficient. ${ }^{35-37}$ In this study, a value of $-2.34\left[\mathrm{~cm}^{-1} / \mathrm{GPa}\right]$ was used, ${ }^{36}$ which is the value for pure $\mathrm{GaN}$ and is close to the value of -2.43 proposed in Ref. 38 . The calculated values of $\sigma_{\text {biaxial }}$ for each layer are shown in Table I.

Using the density-functional theory, the stress coefficient for AlN was calculated previously and was found to be similar to that of $\mathrm{GaN}$, with values of -2.22 and -2.06 reported in Refs. 39 and 40, respectively. The experimentally (a)

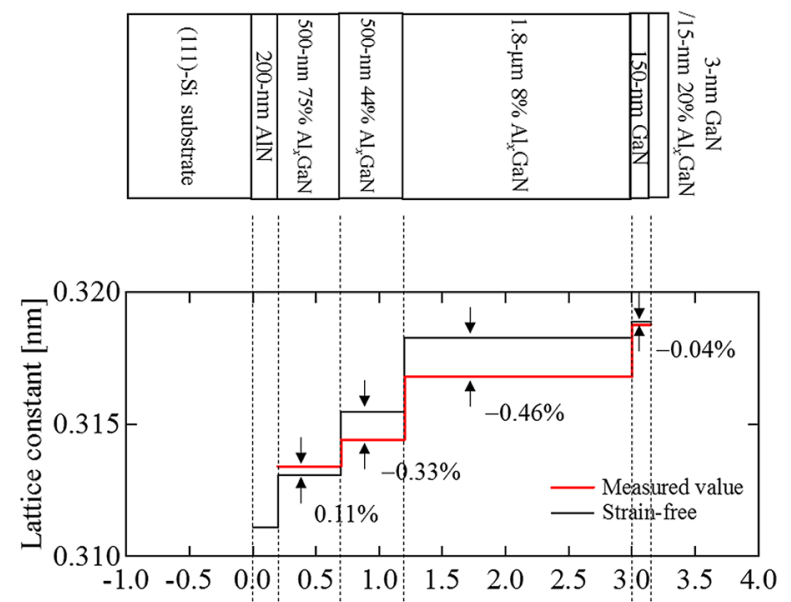

(b)

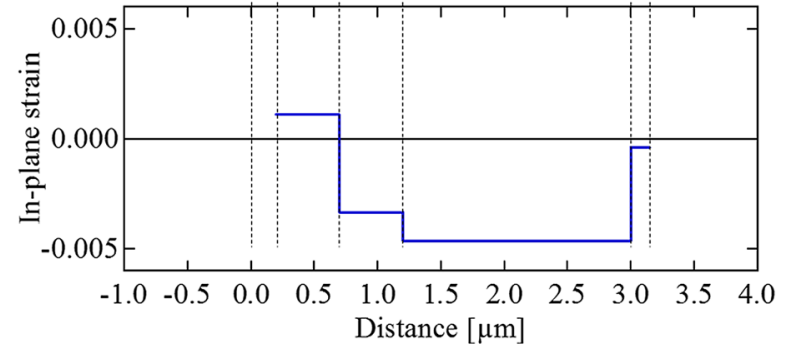

FIG. 4. (a) Lattice constants $a$ of hexagonal $\mathrm{GaN}$ and $\mathrm{Al}_{x} \mathrm{GaN}$ for each layer, with the strain-free lattice constants shown for comparison. (b) Distribution of in-plane strain with depth for the AlGaN/GaN stack.

determined values appear to be slightly smaller than the calculated values. ${ }^{40-44}$ The following discussion is regarded to be qualitatively valid. $\operatorname{as}^{45}$

Using Hooke's law, the in-plane strain $\varepsilon_{\|}$is calculated

$$
\sigma_{\text {biaxial }}=\left(C_{11}+C_{12}-\frac{2 C_{13}^{2}}{C_{33}}\right) \times \varepsilon_{\|},
$$

where $C_{i j}$ is the stiffness constant and the number of independent components for $C_{i j}$ of a wurtzite structure is six: $C_{11}=390, C_{33}=398, C_{44}=105, C_{66}=123, C_{12}=145$, and $C_{13}=106$ for $\mathrm{GaN}$; and $C_{11}=411, C_{33}=389, C_{44}=125$, $C_{66}=131, C_{12}=149$, and $C_{13}=99$ for AlN, with the units as GPa. ${ }^{46,47}$ To obtain $C_{i j}$ for $\mathrm{Al}_{x} \mathrm{Ga}_{1-x} \mathrm{~N}$, we adopted Vegard's law. The calculated in-plane strains $\varepsilon_{\|}$for each layer are shown in Table I.

The lattice constants $a$ of the hexagonal $\mathrm{GaN}$ and $\mathrm{Al}_{x} \mathrm{GaN}$ layers were calculated from the obtained in-plane strains, as shown in Fig. 4(a), with the strain-free lattice constants shown for comparison. Fig. 4(b) shows the distribution of in-plane strain with depth for the AlGaN/GaN stack. Several notable features can be observed. First, the strain in

\begin{tabular}{|c|c|c|c|c|c|}
\hline Material & Raman shift at RT $\left[\mathrm{cm}^{-1}\right]$ & $\omega_{0}^{\mathrm{RT}}$ (Ref. 20) $\left[\mathrm{cm}^{-1}\right]$ & $\Delta \omega\left[\mathrm{cm}^{-1}\right]$ & $\sigma_{\text {biaxial }}[\mathrm{GPa}]$ & $\varepsilon_{\|} \times 10^{-3}$ \\
\hline $\mathrm{GaN}$ & $734.45 \pm 0.19$ & 734 & $+0.45 \pm 0.19$ & $-0.19 \pm 0.08$ & $-0.4 \pm 0.17$ \\
\hline $8 \% \mathrm{Al}_{x} \mathrm{GaN}$ & $756.99 \pm 0.18$ & 751.76 & $+5.23 \pm 0.18$ & $-2.24 \pm 0.08$ & $-4.6 \pm 0.16$ \\
\hline $44 \% \mathrm{Al}_{x} \mathrm{GaN}$ & $823.65 \pm 0.29$ & 819.80 & $+3.85 \pm 0.29$ & $-1.65 \pm 0.12$ & $-3.3 \pm 0.25$ \\
\hline $75 \% \mathrm{Al}_{x} \mathrm{GaN}$ & $861.52 \pm 0.42$ & 862.81 & $-1.29 \pm 0.42$ & $0.55 \pm 0.17$ & $1.1 \pm 0.36$ \\
\hline
\end{tabular}

TABLE I. In-plane strain $\varepsilon_{||}$calculation from the $\mathrm{A}_{1}$ (LO) Raman wavenumber shifts for $\mathrm{GaN}$ and $\mathrm{Al}_{x} \mathrm{GaN}$ layers. 
$75 \% \mathrm{Al}_{x} \mathrm{GaN}$ is relatively small despite the fact that the lattice mismatch between the (111)-oriented Si substrate and $75 \% \mathrm{Al}_{x} \mathrm{GaN}$ is approximately $18 \% .^{48}$ This indicates that the strain in the $\mathrm{AlN}$ and $75 \% \mathrm{Al}_{x} \mathrm{GaN}$ layers may be significantly relaxed. Second, for the $44 \%$ and $8 \% \mathrm{Al}_{x} \mathrm{GaN}$ layers, relatively large compressive strains of $-0.33 \%$ and $-0.46 \%$, respectively, are present. These compressive strains may arise because of the different lattice parameters of these layers than that of the relaxed $75 \% \mathrm{Al}_{x} \mathrm{GaN}$ layer.

In the GaN channel layer, the strain is relaxed to $-0.04 \%$ despite a large difference in lattice spacing with the strained $8 \% \quad \mathrm{Al}_{x} \mathrm{GaN}$ layer. An average strain value of $-0.46 \%$ for the $8 \% \mathrm{Al}_{x} \mathrm{GaN}$ layer is found across the entire layer thickness of $1.8 \mu \mathrm{m}$ although there may be a large gradient in strain with depth. The top portion of the $8 \% \mathrm{Al}_{x} \mathrm{GaN}$ layer can be considered to be more relaxed. Moreover, the lattice mismatch between strain-free $8 \% \mathrm{AlGaN}$ and $\mathrm{GaN}$ is small; therefore, it is considered that the strain in the GaN channel layer was small.

The obtained in-plane strain values shown in Fig. 4(b) include errors to some extent, because of the inaccuracy associated with the strain-free peak positions of $\mathrm{Al}_{x} \mathrm{GaN}$ and the unknown isotropic biaxial stress coefficients for $\mathrm{Al}_{x} \mathrm{GaN}$. If there is an error of $\pm 0.5 \%$ in the $\mathrm{Al}$ concentration, the in-plane strain errors are calculated to be $-4.6 \pm 0.96,-3.3$ \pm 0.70 , and $-1.1 \pm 0.49 \times 10^{-3}$ for $8 \%, 44 \%$, and $75 \%$ $\mathrm{Al}_{x} \mathrm{GaN}$, respectively. From this error analysis, we still think that the above-mentioned discussion is valid. On the other hand, it is considered that the biaxial stress coefficients between GaN and AlN are close, because their stiffness coefficients are close ${ }^{46,47}$ In this respect, the biaxial stress coefficients of $\mathrm{Al}_{x} \mathrm{GaN}$ do not seem to differ much from those of $\mathrm{GaN}$ and AlN. Even if there is a difference in the coefficients between $\mathrm{GaN}$ and $\mathrm{Al}_{x} \mathrm{GaN}$, the discussion described above is qualitatively valid, as mentioned previously.

As seen in Fig. 4, there are large strain variations in the AlGaN stack. This may contribute to the observed differences in temperature dependence, especially for the $\mathrm{E}_{2}$ high mode, as shown in Fig. 3. As mentioned earlier, the $\mathrm{E}_{2}$ high mode is polarized parallel to the sample surface, whereas the $\mathrm{A}_{1}$ (LO) mode is polarized perpendicularly. Therefore, we expect the $\mathrm{E}_{2}$ high mode to be more sensitive to in-plane strain in the AlGaN stack layers. The differences in the calculated temperature dependence of Raman shifts for each layer correlate with the in-plane strain, which was determined by analyzing the spectra of each $\mathrm{AlGaN}$ stack layer simultaneously.

\section{CONCLUSIONS}

In this study, we have analyzed the temperature dependence of Raman shifts for different layers and different optical phonon modes in an AlGaN/GaN stack for HEMTs. The phonon information from the $\mathrm{GaN}$ channel layer and $8 \%$, $44 \%$, and $75 \% \mathrm{Al}_{x} \mathrm{GaN}$ buffer layers was included in a single Raman spectrum, which enabled us to determine the temperature dependence of the $\mathrm{E}_{2}$ high and $\mathrm{A}_{1}$ (LO) modes for each layer simultaneously. Differences in the slopes obtained for each layer were particularly visible in the $\mathrm{E}_{2}$ high mode with a polarization parallel to the sample surface, compared with the perpendicularly polarized $\mathrm{A}_{1}(\mathrm{LO})$ mode. To understand this phenomenon, the in-plane stains induced in the $\mathrm{AlGaN}$ stack were calculated from the $\mathrm{A}_{1}$ (LO) Raman wavenumber shift at RT using the isotropic biaxial stress coefficient. Relatively large compressive in-plane strains were calculated for the 8 and $44 \% \mathrm{Al}_{x} \mathrm{GaN}$ layers, whereas the $\mathrm{GaN}$ channel and $75 \% \mathrm{Al}_{x} \mathrm{GaN}$ layers had significantly relaxed in-plane strains. It is considered that this strain distribution with depth correlates with the differences in temperature dependence of the optical phonons in the $\mathrm{AlGaN} / \mathrm{GaN}$ stack.

\section{ACKNOWLEDGMENTS}

This study was supported by the JSPS Postdoctoral Fellowships for Research Abroad.

${ }^{1}$ V. Sodan, D. Kosemura, S. Stoffels, H. Oprins, M. Baelmans, S. Decoutere, and I. De Wolf, IEEE Trans. Electron Devices 63, 2321 (2016).

${ }^{2}$ C. Hodges, J. A. Calvo, S. Stoffels, D. Marcon, and M. Kuball, Appl. Phys. Lett. 103, 202108 (2013).

${ }^{3}$ A. Sarua, H. Hi, M. Kuball, M. J. Uren, T. Martin, K. P. Hilton, and R. S. Balmer, IEEE Trans. Electron Devices 53, 2438 (2006).

${ }^{4}$ M. Kuball, F. Demangeot, J. Frandon, M. A. Renucci, J. Massies, N. Grandjean, R. L. Aulombard, and O. Briot, Appl. Phys. Lett. 73, 960 (1998).

${ }^{5}$ A. Sarua, H. Ji, M. Kuball, M. J. Uren, T. Martin, K. J. Nash, K. P. Hilton, and R. S. Balmer, Appl. Phys. Lett. 88, 103502 (2006).

${ }^{6}$ C. Hodges, J. Pomeroy, and M. Kuball, J. Appl. Phys. 115, 064504 (2014).

${ }^{7}$ T. Azuhata, T. Sota, K. Suzuki, and S. Nakamura, J. Phys.: Condens. Matter 7, L129 (1995).

${ }^{8}$ D. Shimada, T. Sota, and K. Suzuki, J. Appl. Phys. 84, 4951 (1998).

${ }^{9}$ H. Harima, J. Phys.: Condens. Matter 14, R967 (2002).

${ }^{10}$ V. Yu. Davydov, N. S. Averkiev, I. N. Goncharuk, D. K. Nelson, I. P. Nikitina, A. S. Polkovnikov, A. N. Smirnov, and M. A. Jacobson, J. Appl. Phys. 82, 5097 (1997).

${ }^{11}$ C. Kisielowski, J. Krüger, S. Ruvimov, T. Suski, J. W. Ager III, E. Jones, Z. Liliental-Weber, M. Rubin, E. R. Weber, M. D. Bremser, and R. F. Davis, Phys. Rev. B 54, 17745 (1996).

${ }^{12}$ J. F. Muth, J. H. Lee, I. K. Shmagin, R. M. Kolbas, H. C. Casey, Jr., B. P. Keller, U. K. Mishra, and S. P. DenBaars, Appl. Phys. Lett. 71, 2572 (1997).

${ }^{13}$ S. C. Jain, M. Willander, J. Narayan, and R. Van Overstraeten, J. Appl. Phys. 87, 965 (2000).

${ }^{14}$ R. J. Briggs and A. K. Ramdas, Phys. Rev. B 13, 5518 (1976).

${ }^{15}$ T. C. Damen, S. P. S. Porto, and B. Tell, Phys. Rev. 142, 570 (1966).

${ }^{16}$ C. A. Arguello, D. L. Rousseau, and S. P. S. Porto, Phys. Rev. 181, 1351 (1969).

${ }^{17}$ H. Siegle, G. Kaczmarczyk, L. Filippidis, A. P. Litvinchuk, A. Hoffmann, and C. Thomsen, Phys. Rev. B 55, 7000 (1997).

${ }^{18}$ V. Y. Davydov, Y. E. Kitaev, I. N. Goncharuk, A. N. Smirnov, J. Graul, O. Semchinova, D. Uffmann, and R. A. Evarestov, Phys. Rev. B 58, 12899 (1998).

${ }^{19}$ J. B. Cui, K. Amtmann, J. Ristein, and L. Ley, J. Appl. Phys. 83, 7929 (1998).

${ }^{20}$ V. Y. Davydov, I. N. Goncharuk, A. N. Smirnov, A. E. Nikolaev, W. V. Lundin, A. S. Usikov, A. A. Klochikhin, J. Aderhold, J. Graul, O. Semchinova, and H. Harima, Phys. Rev. B 65, 125203 (2002).

${ }^{21}$ M. Kuball, Surf. Interface Anal. 31, 987 (2001).

${ }^{22}$ T. Azuhata, T. Matsunaga, K. Shimada, K. Yoshida, T. Sota, K. Suzuki, and S. Nakamura, Physica B 219-220, 493 (1996).

${ }^{23}$ A. Link, K. Bitzer, W. Limmer, R. Sauer, C. Kirchner, V. Shwegler, M. Kamp, D. G. Ebling, and K. W. Benz, J. Appl. Phys. 86, 6256 (1999).

${ }^{24}$ W. S. Li, Z. X. Shen, Z. C. Feng, and S. J. Chua, J. Appl. Phys. 87, 3332 (2000).

${ }^{25}$ M. S. Liu, L. A. Bursill, S. Prawer, K. W. Nugent, Y. Z. Tong, and G. Y. Zhang, Appl. Phys. Lett. 74, 3125 (1999).

${ }^{26} \mathrm{Z}$. Guangchen, F. Shiwei, L. Jingwan, Z. Yan, and G. Chunsheng, J. Semicond. 33, 044003 (2012). 
${ }^{27}$ G. Irmer, M. Wenzel, and J. Monecke, Phys. Status Solidi B 195, 85 (1996).

${ }^{28}$ J. Menéndez and M. Cardona, Phys. Rev. B 29, 2051 (1984).

${ }^{29}$ K. Nagata, M. Takei, A. Ogura, and K. Uchida, Jpn. J. Appl. Phys., Part 1 53, 032401 (2014).

${ }^{30}$ R. Sugie, K. Kosaka, H. Seki, H. Hashimoto, and M. Yoshikawa, J. Appl. Phys. 114, 233503 (2013)

${ }^{31}$ M. Balkanski, R. F. Wallis, and E. Haro, Phys. Rev. B 28, 1928 (1983).

${ }^{32}$ H. H. Burke and I. P. Herman, Phys. Rev. B 48, 15016 (1993).

${ }^{33}$ B. C. Johnson, B. Haberl, J. E. Bradby, J. C. McCallum, and J. S. Williams, Phys. Rev. B 83, 235205 (2011).

${ }^{34}$ E. Islam, A. Sakai, and A. Onodera, J. Phys. Soc. Jpn. 70, 576 (2001).

${ }^{35}$ F. Demangeot, J. Frandon, M. A. Renucci, O. Briot, B. Gil, and R. L. Aulombard, Solid State Commun. 100, 207 (1996).

${ }^{36}$ J.-Y. Lu, D.-M. Deng, Y. Wang, K. J. Chen, K.-M. Lau, and T.-Y. Zhang, AIP Adv. 1, 032132 (2011).

${ }^{37}$ A. R. Goni, H. Siegle, K. Syassen, C. Thomsen, and J.-M. Wagner, Phys. Rev. B 64, 035205 (2001).

${ }^{38}$ G. Callsen, J. S. Reparaz, M. R. Wagner, R. Kirste, C. Nenstiel, A. Hoffmann, and M. R. Phillips, Appl. Phys. Lett. 98, 061906 (2011).
${ }^{39}$ J.-M. Wagner and F. Bechstedt, Appl. Phys. Lett 77, 346 (2000).

${ }^{40}$ G. Callsen, M. R. Wagner, J. S. Reparaz, F. Nippert, T. Kure, S. Kalinowski, A. Hoffmann, M. J. Ford, M. R. Phillips, R. F. Dalmau, R. Schlesser, R. Collazo, and Z. Sitar, Phys. Rev. B 90, 205206 (2014).

${ }^{41}$ M. Kuball, J. M. Hayes, A. D. Prins, N. Van Uden, D. J. Dunstan, Y. Shi, and J. H. Edgar, Appl. Phys. Lett. 78, 724 (2001).

${ }^{42}$ F. J. Manjón, D. Errandonea, A. H. Romero, N. Garro, J. Serrano, and M. Kuball, Phys. Rev. B 77, 205204 (2008).

${ }^{43}$ J. Gleize, M. A. Renucci, J. Frandon, E. Bellet-Amalric, and B. Daudin, J. Appl. Phys. 93, 2065 (2003).

${ }^{44}$ W. Zhu, A. Leto, K.-y. Hashimoto, and G. Pezzotti, J. Appl. Phys. 112, 103526 (2012).

${ }^{45}$ M. R. Laskar, T. Ganguli, A. A. Rahman, A. Mukherjee, N. Hatui, M. R. Gokhale, and A. Bhattacharya, J. Appl. Phys. 109, 013107 (2011).

${ }^{46}$ L. E. McNeil, M. Grimsditch, and R. H. French, J. Am. Ceram. Soc. 76, 1132 (1993).

${ }^{47}$ A. Polian, M. Grimsditch, and I. Grzegory, J. Appl. Phys. 79, 3343 (1996).

${ }^{48}$ S. Q. Zhou, A. Vantomme, B. S. Zhang, H. Yang, and M. F. Wu, Appl. Phys. Lett. 86, 081912 (2005). 